\title{
MEASURING CMCCON02 COLOR INCONSTANCY INDEX: USE OF CONSUMER Digital CAMERA AS A SUBSTITUTE FOR REFLECTANCE SPECTROPHOTOMETER
}

\author{
Mahdi Safi ${ }^{1,2}$, Tayebe Soleymanian ${ }^{2,1}$, Keivan Ansari ${ }^{1,3}$ and yannick \\ benezeth $^{3}$ \\ ${ }^{1}$ Deptartment Color Imaging and Color Image Processing, Institute \\ for Color Science and Technology, Tehran, Iran \\ ${ }^{2}$ Deptartment. of Textile Engineering Amirkabir University of \\ Technology,Tehran, Iran \\ ${ }^{3}$ ImViA- Imaging and Artificial Vision,Université de Bourgogne, Dijon, France
}

\begin{abstract}
Today, many attempts have been made to use the digital color cameras for color measurement applications. The gold of this work is a comparison between the measure of CMCCONO2 color inconstancy index obtained by the spectrophotometric data and the data from a characterized digital color camera. According to the results, the square of correlation coefficient of 0.8 was obtained between the color inconstancy indices acquired of the spectrophotometric data and the camera RGB data. The attained differences between two methods could be decreased by improving camera characterization process. Besides, it is observed the spectral power distribution of the applied illuminant lead to the important effects on the results of colorimetric characterization of the camera.
\end{abstract}

\section{KEYWORDS}

Color inconstancy index, CMCCON02, Digital camera, Spectrophotometer, Characterization, White balance.

\section{INTRODUCTION}

Color of an object which measured by image capturing apparatus like scanner and camera can take different color values under different light sources [1,2]. It can be considered in terms of color inconstancy index. Therefore reciprocally, color constancy is a visual effect where colors remain relatively uniform under different light sources conditions [3]. In fact, the aim of color constancy is to correct for influence of illuminant color on object color [4]. Generally, colorimetric data from spectrophotometric measurements, i.e. tristimulus values (XYZ) under test and reference illuminants are applied to calculate color inconstancy index (CII). For numerical evaluation of color constancy, two important color inconstancy indexes, i.e. CMCCON97 and CMCCON02 are developed [2]. The CMCCON97 index was proposed by Luo and Hunt in 1997 based on CMCCAT97 which can accurately predict the corresponding colors. Subsequently, the chromatic adaption transform CMCCAT97 was modified and a new chromatic adaptation transform, CAT02 was introduced [5]. In recent decades, the CMCCON02 index, as the last suggested color inconstancy index has been used to predict the direction and the value of the color variations under the test and the reference illuminants for a standard viewer [6].

Natarajan Meghanathan et al. (Eds) : ICCSEA, WiMoA, SCAI, SPPR, InWeS, NECO - 2019 pp. 175-181, 2019. (C) CS \& IT-CSCP 2019

DOI: $10.5121 /$ csit.2019.91814 
Today, with the growth of digital cameras, it is possible to use the camera as a spectrophotometer replacement for applications such as color reproduction, measuring color of skin, digital computer matching of tooth color and so on. [7-10]. The object of present work was using a digital color camera to measure the CMCCON02 color inconstancy index. The results of this study were compared with the results of spectrophotometer data.

\section{METHOD}

\subsection{Sample Preparation}

The SG color input target with 140 different color samples was selected. An Eye-One spectrophotometer with 45:0 geometry and a diameter aperture $4 \mathrm{~mm}$ was selected to read the spectral data of the samples. The reflectance values were measured at $10 \mathrm{~nm}$ intervals from 400 to $700 \mathrm{~nm}$. Additionally, a CANON EOS 500D camera was used for the acquisition of color images. The captured images in two modes of white balance ON and OFF were saved as RAW and JPEG image formats. All the photography were performed in a VeriVide light booth under illuminant D65 and A, separately (Figure. 1). The specifications of the employed illuminants are reported in Table 1 and Figure 2.

As Figure 1 shows to prevent mirror reflection, all the photography were performed at 45:0 lighting geometry. Besides, the lighting homogeneity was controlled by the proposed method in the mentioned reference [11].

\subsection{Experimental Procedure}

The tristimulus values of the samples were calculated under CIE standard illuminants D65 and A and the 1964 standard observer and consequently the color inconstancy index was determined according to the CMCCON02 as a known color adaptation algorithm as follows [6]:

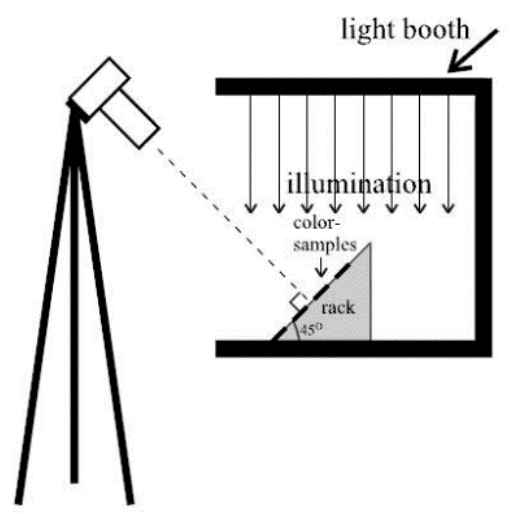

Figure. 1. The geometry of color image acquisition.

- Calculation of the XYZ of the sample under the D65 illuminant and under the test illuminant (X, Y, Z).

- Determination of camera responses of the sample $(\mathrm{R}, \mathrm{G}, \mathrm{B})$ and the reference under the test illuminant $\left(\mathrm{R}_{\mathrm{W}}, \mathrm{G}_{\mathrm{W}}, \mathrm{B}_{\mathrm{W}}\right)$ and the D65 illuminant $\left(\mathrm{R}_{\mathrm{Wr}}, \mathrm{G}_{\mathrm{Wr}}, \mathrm{B}_{\mathrm{Wr}}\right)$.

- Calculating the corresponding RGB cone responses.

- Determination of the XYZ of the corresponding color under the D65 illuminant.

The magnitude of color inconstancy was defined by Delta LAB $\left(\Delta E_{a b}^{*}\right)$. Besides, a colorimetric characterization method was set to convert RGB values to CIEXYZ by a nonlinear regression 


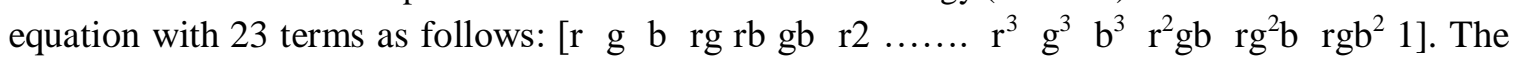
colorimetric characterization error for the training and the testing samples was calculated in terms of Delta LAB unit. The square correlation coefficient $\left(\mathrm{R}^{2}\right)$ for the predicted color coordinates with subtitle $\mathrm{P}$ and the actual values is plotted in Figure 3.

Table 1 . The chromaticity coordinates of the applied illuminants D65 and A.

\begin{tabular}{|l|l|l|}
\hline Illuminant & D65 & A \\
\hline $\mathrm{x}$ value & 0.31 & 0.45 \\
\hline $\mathrm{y}$ value & 0.33 & 0.40 \\
\hline
\end{tabular}

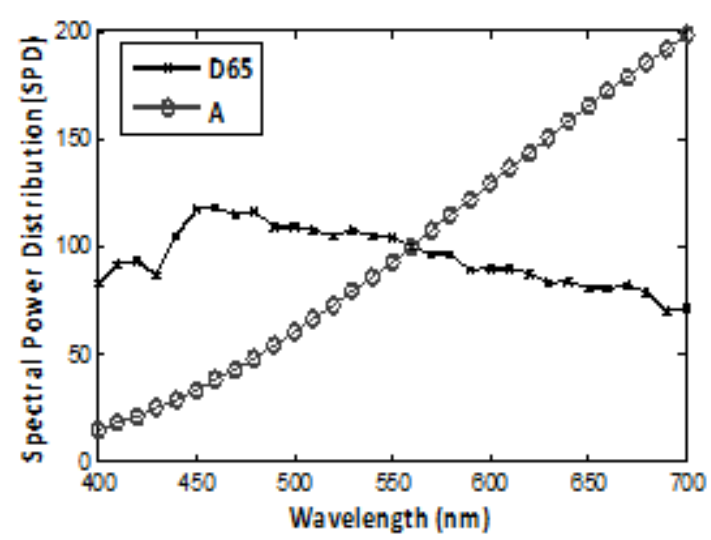

Figure 2. The SPD of the applied illuminants D65 and A.
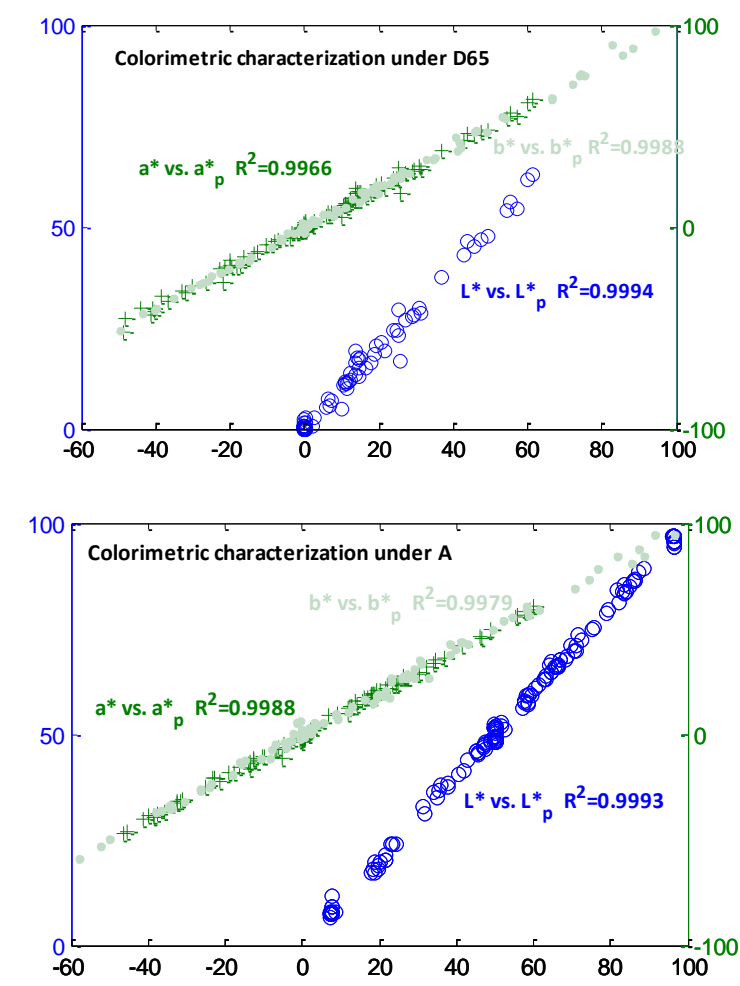

Figure 3. $\mathrm{LP}^{*}$ vs. $\mathrm{L}^{*}$, aP* vs. $\mathrm{a}^{*}$, and $\mathrm{bP}^{*}$ vs. $\mathrm{b}^{*}$ under D65 and A illuminant 


\section{RESUlts AND DisCUSSION}

The color inconstancy index of 140 samples was obtained from the spectrophotometric data using CAT02 transform. The results are plotted in Figure 4 against the hue angle of the samples. The obtained results show that the samples of SG color input target behave in different degrees of color constancy ranged between 0.09-14.8 of color inconstancy index. The charcterizing performance to convert RGB values to CIEXYZ by using for both RAW and JPEG image formats as well as white balance ON and OFF modes are reported in Table 2.

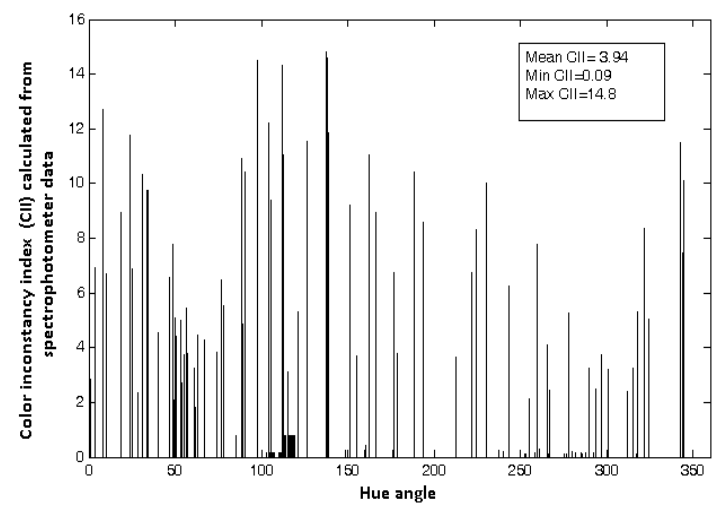

Figure 4. The color inconstancy index calculated vs. hue angle obtained from the spectrophotometric data.

From Table 2, the minimum error was obtained for the RAW image format in white balance ON mode. According to the results for the RAW image format in white balance ON mode, the characterization error value for the training and the testing steps under illuminant D65 and illuminant A was obtained 0.91, 1.46, 1.32 and 1.90, respectively. It seems the spectral power distribution of light source has the significant effect on characterizing performance. Consequently, it can be seen from TABLE 2 that illuminant D65 benefits of smaller characterization error value in comparision with illiuminant $\mathrm{A}$.

At the next step, a RAW image with considering white balance $\mathrm{ON}$ was selected to estimate the CIEXYZ values and the color inconstancy index. The color difference by Delta LAB between two images captured under illuminant D65 and illuminant A was considered as color inconstancy index without usage of color inconstancy algorithm. In fact, it was tried to study the effect of white balance as substitutes for color inconstancy index. The results are plotted in Fig. 5 against the color inconstancy index vlues obtained from the spectrophotometric data. As Fig.5 shows, the acquired agreement is poor with an approximately $30 \%$ correlation between the results. In other words, a RAW image with considering white balance ON can just retrieval a small part of the CMCCON02 color inconstancy algorithm's results. Then, the CAT02 algorithm was applied to estimate the color inconstancy index. The results are illustrated in Fig. 6. An $R$-squared equals 0.8 shows a relatively better agreement than the previous results in Fig. 5. Moreover, Fig.7 illustrates the difference between the color inconstancy index values estimated from the camera and the spectrophotometer dada for the selected samples.

Evidently, the error of camera characterization which involved in the conversion of RGB to XYZ, is considered to be an unavoidable error source for the acquired differences. The samples with low lightness could be considred as another source of error. Accordingly, it seems the applied regression of characterization method wasn't able to estimate the corrected color for the samples under changing the illuminant at low levels of lightness, say $L^{*}<50$, like the black ones. Consequently, it is resulted in estimating of color inconstancy index with less accuracy. 
Computer Science \& Information Technology (CS \& IT)

Table 2. The performance of characterization in terms of mean error in the different conditions.

\begin{tabular}{|c|c|c|c|c|c|}
\hline \multirow{2}{*}{} & \multicolumn{5}{|c|}{ Mean Error (Delta LAB) } \\
\cline { 2 - 6 } & Step & $\begin{array}{c}\text { RAW } \\
\text { W.B.:ON }\end{array}$ & $\begin{array}{c}\text { RAW } \\
W . B .: O F F\end{array}$ & $\begin{array}{c}\text { JPEG } \\
W . B .: O N\end{array}$ & $\begin{array}{c}\text { JPEG } \\
\text { W.B.:OFF }\end{array}$ \\
\hline D65 & Training & 0.91 & 1.06 & 1.80 & 20.50 \\
\hline D65 & Testining & 1.46 & 1.69 & 3.53 & 21.91 \\
\hline A & Training & 1.32 & 1.78 & 4.66 & 29.68 \\
\hline A & Testing & 1.90 & 2.79 & 9.08 & 29.26 \\
\hline
\end{tabular}

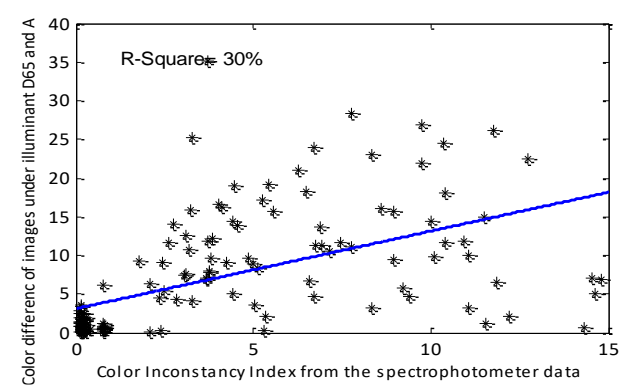

Figure 5. The estimated color inconstancy index from the camera data vs. those from the spectrophotometric data: color inconstancy algorithm is NOT applied.

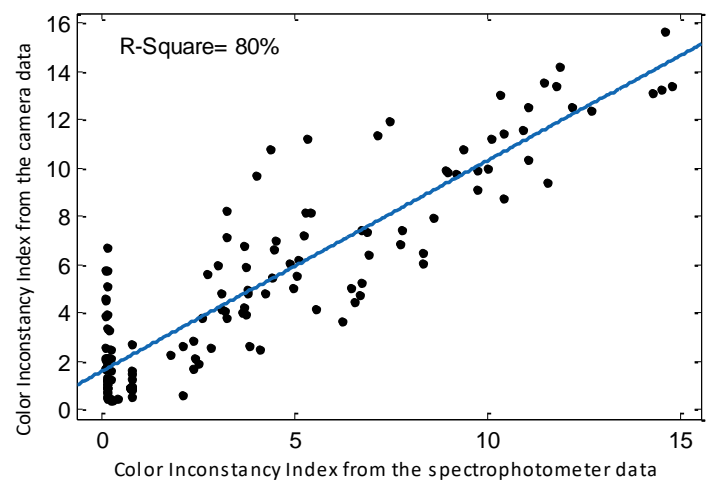

Figure 6. The estimated color inconstancy index from the camera data vs. those from the spectrophotometric data: color inconstancy algorithm is applied.

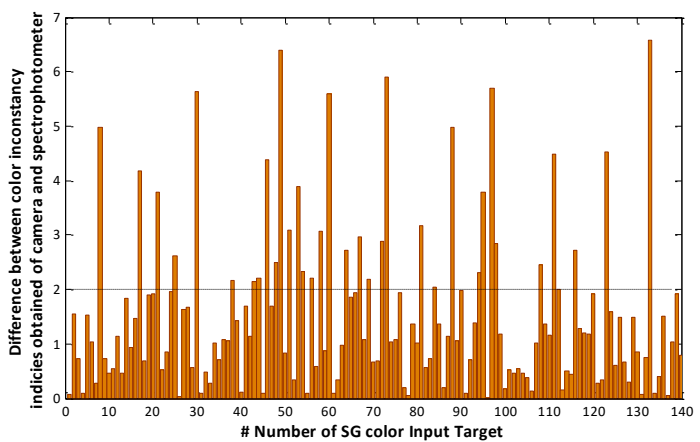

Figure 7. The difference between color inconstancy index values obtained from the camera and the spectrophotometric data. 


\section{Conclusions}

In this study, a digital color camera was applied to estimate the CMCCONO2 color inconstancy index under the illuminant pair of D65-A. The camera was characterized by a nonlinear regression equation. The best result of characterization was obtained for the RAW images and the white balance ON mode. According to the acquired results, illuminant A and D65 showed a specific difference on the characterizing performance. It observed that it was affected by the value of spectral dissimilarity of the applied illuminants. Besides, the square of correlation coefficient of 0.8 was obtained between the color inconstancy index values from the camera and the spectrophotometric data. It was concluded that the acquired deviation is related to the error of the characterization process, as it is usually a non-removable parameter. Furthermore, the lightness level of the sample plays an important role that must be considered.

\section{REFERENCES}

[1] S. Aulakh and A. Arora, (2013) "A Review on Color Constancy Algorithms," International Journal of Engineering and Innovative Technology, vol. 3, pp. 180-183.

[2] M. Safi, (2014) "A Study of Relationship between Color Inconstancy Level and CIELAB Color Coordinates",Prog. Color Colorants Coat., vol. 7, pp. 165-175.

[3] H. R. Kang,( 2006) Computational color technology, SPIE press.

[4] L. T. Maloney, B. A. Wandell, (1986) "Color constancy: a method for recovering surface spectral reflectance," J. Opt. Soc. Am. A, vol. 3, pp. 29-33.

[5] M. R. Luo, R. W. G. Hunt, B. Rigg, K. J. Smith,( 1999) "Recommended color-inconstancy index," J. Soc. Dyers. Color., vol. 115, 183-188.

[6] "Textiles - tests for color fastness, part J05, method for the instrumental assessment of the color inconstancy of a specimen with change in illuminant (CMCCONO2)". ISO 2007.

[7] F. D. Jarad, M. D. Russell and B.W. Moss, (2005) "The use of digital imaging for colour matching and communication in restorative dentistry," British dental Journal, vol. 199, pp. 43-49.

[8] K. S. Bersh, (2010) "Spectral imaging and analysis of human skin," Master Thesis, University of Eastern Finland.

[9] A. Verikas, "Estimating ink density from colour camera RGB values by the local kernel ridge regression, Engineering Applications of Artificial Intelligence, 21, pp. 35-42, 2008.

[10] C. Thorstenson, (2017) "Validation of a Method to Estimate Skin Spectral Reflectance Using a Digital Camera", Master Thesis, Rochester Institute of Technology.

[11] A. Mahmoudi, M. Safi, (2014) "Investigating and Proving of Multiplicative Lighting Correction in Color Characterization of Digital Camera," J. Color Sci. Tech, vol. 8, pp. 109-115. 
Authors

Mahdi Safi received the B.S., M.S. and Ph.D. degrees in textile chemistry from Amirkabir University of Technology, Tehran, Iran, in 1999, 2001 and 2008, respectively. He is currently an assistant professor of color science in the Department of Color Physics, Institute for Color Science and Technology, Tehran, Iran. His research interests include colorimetry, light source, displays, color difference, color matching.

Tayebe Soleymanian received the B.S., M.S. degrees in textile chemistry from Amirkabir University of Technology, Tehran, Iran, in 2014 and 2016, respectively. She is currently a $\mathrm{PhD}$ student in textile chemistry in the Department of Textile Engineering, Amirkabir University of Technology, Tehran, Iran. Her research interests include colorimetry, color imaging, displays.

Keivan Ansari received his Ph.D. in color engineering from Amirkabir University (Polytechnic of Tehran),2005. He is an assistant professor in Color Imaging \& Color Image Processing research group in Color for Science \& Technology Institute, Tehran, IRAN. He is currently pursuing his postdoctoral at the ImViA laboratory in the Bourgandy university, Dijon, FRANCE. His work has focused on the development of Color Physics and its application in image processing.

Yannick Benezeth is associate professor at the Univ. Bourgogne Franche-Comté (France). He obtained his Ph.D. in computer science from the Univ. of Orléans in 2009. He also received the engineering degree from the ENSI de Bourges and the MS degree from the University of Versailles-Saint-Quentin-en-Yvelines in 2006. His research interests include biomedical engineering, image processing, and video analytics. Application areas include video health monitoring and endoscopy.
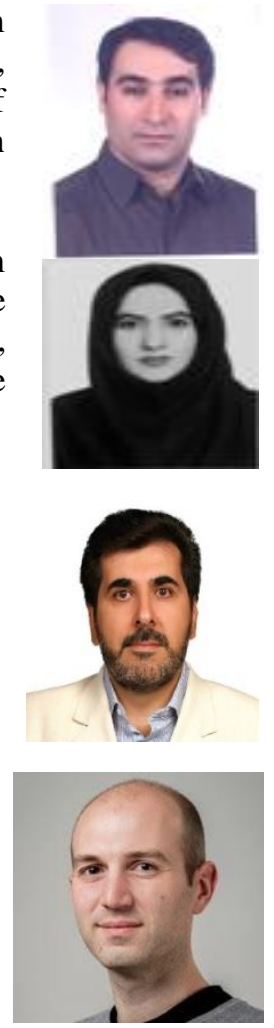